\title{
Sustainable Urban Mobility Boost Smart Toolbox
}

DOI: https://doi.org/10.7307/ptsm.2020.6

\author{
Marko Šoštarić ${ }^{1}$, Marijan Jakovljević ${ }^{1}$, Orsat Lale ${ }^{1}$, Krešimir Vidović ${ }^{2}$, Saša Vojvodić ${ }^{2}$ \\ University of Zagreb Faculty of Transport and Traffic Sciences ${ }^{1}$; Ericsson Nikola Tesla ${ }^{2}$
}

\author{
Keywords: \\ Big data \\ Transport \\ planning \\ Commuter pattern \\ Urban mobility
}

\begin{abstract}
Traffic system analysis and planning is a very complex process that requires quality input data collected on a relevant sample and over a relevant time period. The project Sustainable Urban Mobility Boost Smart Toolbox aims at development of the methodology (toolbox) in data rich reality, which is combining traditional and novel data science approach for transport system analysis and planning. It enables digital transformation of existing (traditional, ingrained) analytic methodologies by novel utilization of mobile network infrastructure as urban mobility data sources (spatio-temporal data on population migrations gathered from anonymized mobile network logs) and data science capabilities. The project is funded by the EIT Urban Mobility Regional Innovation Scheme RIS 2020.

The end product will provide transport planners with insight in spatial distribution of commuters and their transport means. Also, it will propose methodology for the identification and implementation of the measures for improvement of the transport system based on input data. Primary goal of the Project is to provide universal methodology suitable for any city to create sustainable transport system.
\end{abstract}

\section{Introduction}

In the context of the global problem of the excessive harmful impact of traffic system on the environment, and especially transport in urban centres, the European Union prescribes numerous tenders for the co-financing of scientific projects. One of those is the Urban Mobility Boost Smart Toolbox funded by the EIT Urban Mobility Regional Innovation Scheme RIS 2020.

The toolbox aims at utilizing and combining both traditional and novel methodologies to determine key transport findings and propose alternative, sustainable solutions in urban mobility domain. Traditional methodology involves analysis of spatial characteristics of population migration obtained from classical research (field counting and household/roadside interviewing). Novel methodology will utilize data science approach on telecom originated anonymized big data sets, together with other available datasets for the case study. Proposed methodology will be used to determine specific commuter patterns, and to identify transport demand that is being realized by using ecologically non preferred modes of transport (personal cars). Also, the potential for development of sustainable mobility services in order to address those migrations will be analyzed from various perspectives (infrastructure for sustainable mobility, availability of public transport services etc.)

With a vision of achieving the main goal, the project team made of University of Zagreb Faculty of Transport and Traffic Sciences, Ericsson Nikola Tesla and City of Rijeka developed and described a detailed scheme of a universally applicable toolbox. To demonstrate the operational functionality of the toolbox, the project team selected the City of Rijeka as an adequate city for the conduction of a case study.

The main part of the case study is conducted through big data collecting and traditional field research. The main goal is to validate the big data with the field research results. In the next chapters the process and methods will be described together with the results. 


\section{Methods}

The developed methodology is shown at the Figure 1. The methodology should be able to determine groups with low accessibility to public transport or cycling and walking routes and help the city governments to provide them sustainable mobility modes. The main part of the case study is conducted through big data collecting and traditional field research. The main goal is to validate the big data with the field research results. In the next chapters the process and methods will be described together with the results.

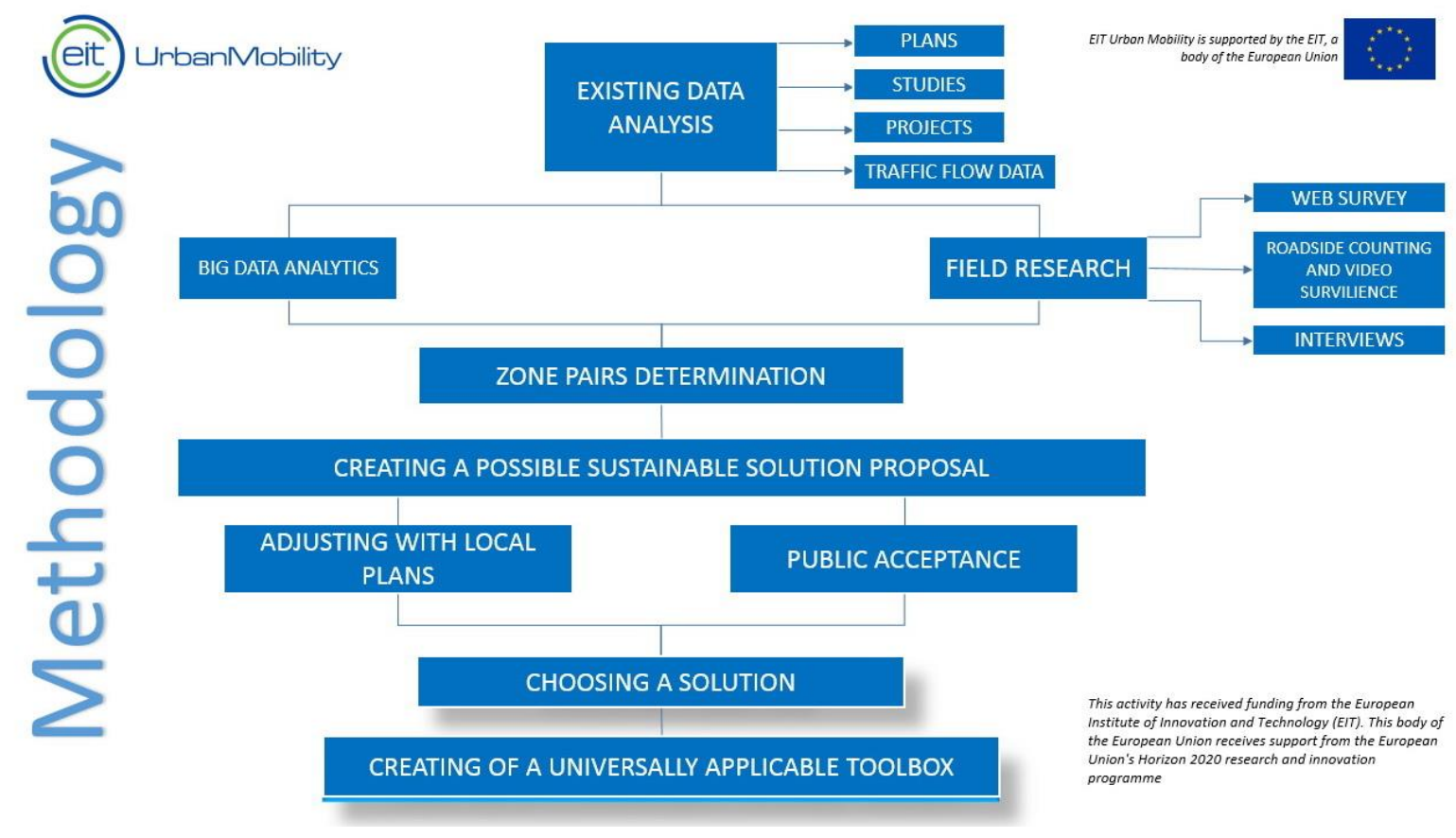

Figure 1. methodology

Desktop research has been done to gain the insight in the transport system and its already identified challenges and future development plans. It was conducted by the Faculty of Transport and Traffic Sciences with the support of the City of Rijeka in providing key transport and planning documentation. Research resulted with description of situation of coverage area regarding economics, demographics, spatial planning, traffic flows and transport infrastructure.

Besides the desktop research, the review of the current transport situation is gained through field research. Field research was performed by an online survey, traffic flow counting and traffic flow spatial distribution. In the survey, citizens of Rijeka were asked about the origin, destination, and travel mode for their usual everyday travel. In an online survey, 1.585 answers were collected. The most significant zones are furthermore analysed by conducting additional telephone interviews. The extra 2.632 responses are collected by phone interviews. For validation of online surveys and interviews, field traffic flow counting and traffic flow spatial distribution analysis were conducted. For this purpose, more than 100.000 vehicles were counted, and 72.000 licence plate numbers collected. Comparison of results gathered by two traditional methods showed great significance with results gathered by online surveys and interviews. 


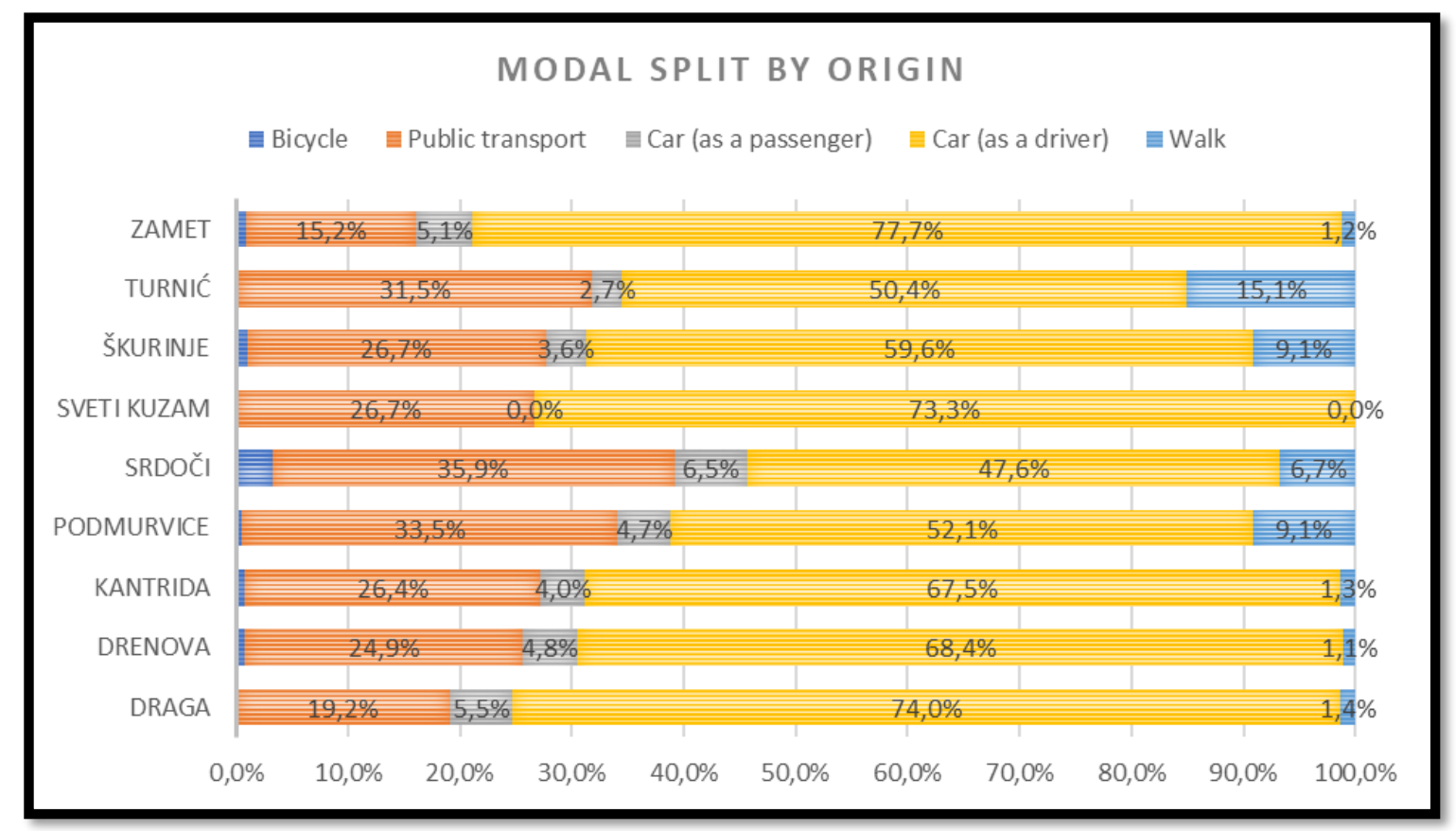

Figure 2. Modal split by travel origin (household survey)

Big data research was performed on anonymised big data sets originating from mobile telecommunication network operator with significant market share of $35 \%$. The total $2.653 \mathrm{~TB}$ of compressed data was gathered by using this method. The data was anonymised and checked for GDPR compliance, and then transferred to Ericsson Nikola Tesla for processing. After performing the first step of data science, the set of 890.101 trips (non pondered value based on 35\% sample) were extracted for broader coverage area and one-week time period. Further analysis was performed on 286.290 (non-pondered value) trips identified for narrower coverage zone (34 zones). The process included development and application of methodology for identification of trips and its characteristics including trip distance, speed, duration, origin, destination, and transport mode for every trip. This analysis resulted in 62.240 (non-pondered value) representative daily trips for characteristic working day.

\section{Results}

Desktop research pointed that City of Rijeka needs a new approach to transport planning and more productive implementation of sustainable solutions. Preliminary analysis of the results of field research managed to obtain similar data on the number of trips of personal cars using two classical research methods and it is expected that the results of field research are an adequate dataset for verification and calibration of big data set.

The comparison of results of traditional and novel big data method was performed on the next parameters: modal split, trip distribution (number of trips between typical zones) and traffic volume. Comparative analysis of results of modal split of travel showed the existence of a strong connection between the results obtained by classical field research in relation to the results obtained by the analysis of a big data sets. The difference between the mentioned methodologies for personal vehicles is $2.49 \%$, public transport $2.56 \%$ and active modes of transport about $0.70 \%$. In accordance with the set assumptions, the average deviation in trip distribution from the analysed zones towards the city center was determined in the amount of about 7.5\% (based on household survey) (Figure 3). 


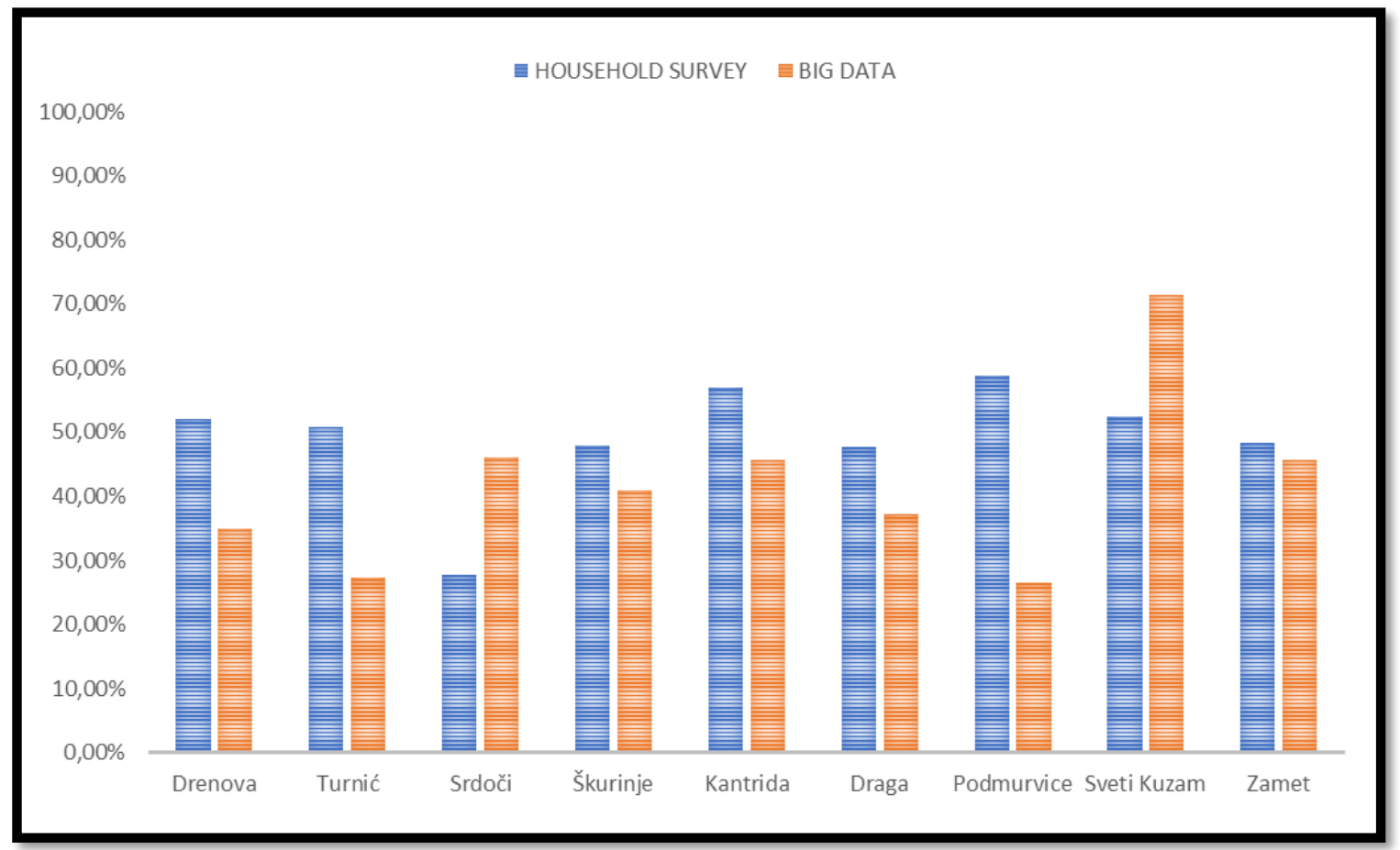

Figure 3. Comparative analysis of travel distribution (household surveys)

A comparative analysis of daily distribution during the characteristic day of the week also revealed the existence of strong connection between the results obtained by classical field research in relation to the results obtained by the analysis of a big data sets $(\mathrm{R} 2=96 \%)$. The results prove that using of presented big data science methods can be appropriate for transport planning and that those methods can support or in some cases replace conventional researches. For total replacement of traditional analysis by big data science, some further studies need to be performed.

Based on big data and traditional analysis, five zones with significant usage of a personal car for daily commuting are determined. The average percentage of personal car usage for daily commuting in City of Rijeka is $72 \%$ while those five zones have a higher percentage. The reason for that increased usage of personal car is mostly the unsuitability or the lack of public transport and the hilly terrain with lack of appropriate footpaths or cycling lanes that restricts the use of bicycle or walking.

\section{Discussion}

During the validation of the big data, it was found that the big data set sometimes provides a more detailed database than required for understanding of transport system. The big data detects all the trips that user makes during the day. It records all type of movement, short trips within the zone or even short recreative walking. That kind of movement are usually not the transport research objective.

\section{Conclusion}

The main results and conclusion are that approach that combines big data science methods with traditional methodologies is appropriate and applicable for transport planning and that the symbiose of those methods can significantly improve conventional researches. In addition to this positive conclusion the project has identified additional potential for big data and data science methodology application in transportation planning, which will be further researched and developed. 
Šoštarić, M., Jakovljević, M., Lale, O., Vidović, K., Vojvodić, S. / Public Transport \& Smart Mobility 2020, 51 55

\section{References}

[1] City of Rijeka, "General Urban Plan of the City of Rijeka”, 2019.

[2] Primorje. Gorski Kotar county "Master Plan for the Development of the Transport System of the Functional Region of North Adriatic", 2018.

[3] Gonçalves, D. N. S., Gonçalves, C. D. M., Assis, T. F. De, \& Silva, M. A. Da. (2014). Analysis of the difference between the euclidean distance and the actual road distance in Brazil. Transportation Research Procedia, 3(July), 876-885.

[4] Buczkowska, S., Coulombel, N., \& Lapparent, M. De. (2016). Euclidean versus network distance in business location: A probabilistic mixture of hurdle-Poisson models. Annals of Regional Science, (964), $21 \mathrm{p}$. 